\title{
Longer term R\&D on Neutrino beams and Neutrino Factories
}

\author{
Ken Peach \\ John Adams Institute for Accelerator Science \\ University of Oxford and Royal Holloway Univerity of London \\ Denys Wilkinson Building, Keble Road, Oxford OX1 3RH, UK \\ E-mail: Ken.Peach@adams-institute.ac.uk
}

Recent developments in neutrino physics, primarily the demonstration of neutrino oscillations in both atmospheric neutrinos and solar neutrinos, provide the first conclusive evidence for physics beyond the Standard Model of particle physics. The phenomenology of neutrino oscillations, for three generations of neutrino, requires six parameters - two squared mass differences, 3 mixing angles and a complex phase that could, if not 0 or $\pi$, contribute to the otherwise unexplained baryon asymmetry observed in the universe. Exploring the neutrino sector will require very intense beams of neutrinos, and will need novel solutions. 


\section{Introduction}

The "Standard Model" of particles and interactions provides an accurate description of huge volumes of data, from LEP, HERA, Tevatron, and experiments like the muon g- 2 . However, convincing evidence [1] for neutrino oscillations, where neutrinos created in a flavour eigenstate (e.g, as $v_{\mathrm{e}}$ in the sun) are subsequently found to be a mixture of flavours, indicates that neutrinos have a mass (however small), while the Standard Model requires the neutrinos to be strictly massless. It is difficult to add a mass term for the neutrinos, in analogy to the quarks and charged leptons, and so neutrino oscillations require physics "beyond the Standard Model".

The 3 flavour eigenstates $\left(v_{e}, v_{\mu}, v_{\tau}\right)$ are related to the 3 mass eigenstates $\left(v_{1}, v_{2}, v_{3}\right)$ through the Maki-Nakagawa-Sakata matrix $\mathrm{U}_{\mathrm{MNS}}$ (equation (1)).

$$
\begin{aligned}
& {\left[v_{e}, v_{\mu}, v_{\tau}\right]=\left[\begin{array}{ccc}
U_{e 1} & U_{e 2} & U_{e 3} \\
U_{\mu 1} & U_{\mu 2} & U_{\mu 3} \\
U_{\tau 1} & U_{\tau 2} & U_{\tau 3}
\end{array}\right]\left[\begin{array}{l}
v_{1} \\
v_{2} \\
v_{3}
\end{array}\right] \quad(1)} \\
& U_{M N S} \equiv\left[\begin{array}{ccc}
\mathrm{c}_{12} & \mathrm{~s}_{12} & 0 \\
-\mathrm{s}_{12} & \mathrm{c}_{12} & 0 \\
0 & 0 & 1
\end{array}\right] \times\left[\begin{array}{ccc}
\mathrm{c}_{13} & 0 & \mathrm{~s}_{13} e^{-i \delta} \\
0 & 1 & 0 \\
-\mathrm{s}_{13} e^{-i \delta} & 0 & \mathrm{c}_{13}
\end{array}\right] \times\left[\begin{array}{ccc}
1 & 0 & 0 \\
0 & \mathrm{c}_{23} & \mathrm{~s}_{23} \\
0 & -\mathrm{s}_{23} & \mathrm{c}_{23}
\end{array}\right] \times\left[\begin{array}{ccc}
e^{i \alpha} & 0 & 0 \\
0 & e^{i \beta} & 0 \\
0 & 0 & 1
\end{array}\right]
\end{aligned}
$$

where $c_{i j}=\cos \theta_{i j}$ and $s_{i j}=\sin \theta_{i j}$.

The frequency of neutrino oscillations is governed by two independent parameters:differences in the squared neutrino masses $-\Delta m_{12}^{2}=m_{2}^{2}-m_{1}^{2}$ and $\Delta m_{23}^{2}=m_{3}^{2}-m_{2}^{2}$. The amplitude of the neutrino oscillations is determined by the appropriate combination of the mixing angles $\theta_{12}, \theta_{23}$, and $\theta_{13}$. The phase $\delta$ violates both $\mathrm{CP}$ and $\mathrm{T}$, and may be related to the baryon asymmetry of the Universe via leptogenesis. The mass scale is determined by measuring the electron neutrino mass or a weighted average mass in neutrinoless double $\beta$ decay, or through astrophysical measurements. The Majorana phases $\alpha$ and $\beta$ play no part in the neutrino oscillations, but they influence neutrinoless double $\beta$ decay. The current values of the parameters [1] are $\Delta m_{12}^{2}=6.9_{-0.40}^{+0.75} \times 10^{-5} \mathrm{eV}^{2}, \theta_{12}=\left(33.2_{-1.6}^{+1.8}\right)^{\circ},\left|\Delta m_{23}^{2}\right|=2.3_{-0.45}^{+0.35} \times 10^{-3} \mathrm{eV}^{2}$,

$$
\theta_{23}=\left(46.1_{-5.0}^{+4.1}\right)^{\circ} \text {, and } \theta_{13}<11^{\circ} \text {; the sign of } \Delta m_{23}^{2} \text { and } \delta, \alpha, \beta \text { are unknown, and } m_{e}<2.1 \mathrm{eV} \text {. }
$$

More intense and better known neutrino beams are required to disentangle the contributions from the different terms governing the oscillation phenomena (see equation (2). The presence of so many trigonometric functions means that there can be several equivalent solutions for the fit to any given distribution, particularly if the statistics are limited.

$$
\begin{aligned}
& P\left(v_{\mu} \Rightarrow v_{e}\right)=4 c_{13}^{2} s_{12}^{2}\left(c_{12}^{2} c_{23}^{2}-s_{12}^{2} s_{13}^{2} s_{23}^{2}-2 c_{12} c_{23} s_{12} s_{23} s_{13} \cos \delta\right) \sin ^{2}\left(\frac{\Delta m_{21}^{2} L}{4 E}\right) \\
& +8 c_{13}^{2} s_{12} s_{13} s_{23}\left(c_{12} c_{23} \cos \delta-s_{12} s_{13} s_{23}\right) \cos \left(\frac{\Delta m_{32}^{2} L}{4 E}\right) \sin \left(\frac{\Delta m_{31}^{2} L}{4 E}\right) \sin \left(\frac{\Delta m_{21}^{2} L}{4 E}\right) \\
& +4 c_{13}^{2} s_{13}^{2} s_{23}^{2} \sin ^{2}\left(\frac{\Delta m_{13}^{2} L}{4 E}\right)-8 c_{13}^{2} c_{12} c_{23} s_{12} s_{13} s_{23} \sin \delta \sin \left(\frac{\Delta m_{32}^{2} L}{4 E}\right) \sin \left(\frac{\Delta m_{31}^{2} L}{4 E}\right) \sin \left(\frac{\Delta m_{21}^{2} L}{4 E}\right)
\end{aligned}
$$




\section{Neutrino Beams and Experiments running or under construction}

So far, the evidence for neutrino oscillations from conventional horn-focussed accelerator derived neutrino beam comes from the LSND experiment [2] and the K2K experiment [3]. K2K produced evidence for $v_{\mu}$ oscillations consistent with the atmospheric neutrino oscillation data. The LSND result is controversial, and should be either confirmed (which would be a major discovery) or refuted by the MiniBooNE experiment [4] underway at Fermilab.

A new neutrino beam (NuMI - Neutrinos at the Main Injector) started running at Fermilab earlier this year [5]. This is a conventional, but high power $(0.3 \mathrm{MW})$, design with two magnetic horns which can, by reconfiguring, produce neutrino beams with different peak energies, directed towards a detector $735 \mathrm{~km}$ distant, at the Soudan mine in Minnesota. Even though the target mass is only about $20 \%$ of that of $\mathrm{K} 2 \mathrm{~K}$, the event rate in MINOS is about 30 times that of $\mathrm{K} 2 \mathrm{~K}$. The principal physics goals of MINOS are a precision measurement of the atmospheric $\left(v_{\mu}\right.$ or " 23 ") oscillation parameters, and to improve the limits on (or make a measurement of)

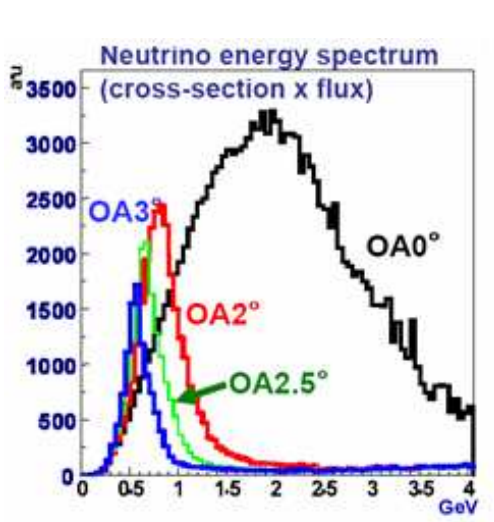

Figure 1 The "off-axis" trick $\theta_{13}$. The "CERN to Gran Sasso" (CNGS) beam is also a conventional neutrino beam which, unlike the $\mathrm{K} 2 \mathrm{~K}$ and NUMI beams, is at a relatively high energy (above the threshold for production of $v_{\tau}$ ). This will start commissioning in 2006. The main objective is to demonstrate $v_{\mu} \rightarrow v_{\tau}$ appearance.

So far, neutrino beams have been "on-axis"; this gives the highest flux of neutrinos, but inevitably has a broad momentum spectrum, with a long high energy tail, even for the so-called "narrow-band beams". Because of the small Qvalue in $\pi$-decay, the neutrino energy spectrum off-axis [6] has a narrower momentum spread, smaller high energy tail and higher flux at the peak energy, than the on-axis beam (see Figure 1). The "Tokai to Kamiokande" (T2K) experiment [7] now under construction at J-PARC uses this feature, combined with a high-energy $(50 \mathrm{GeV})$, high power $(0.75 \mathrm{MW}$, upgradeable to $4 \mathrm{MW})$ proton beam to produce the first long-baseline $(295 \mathrm{~km})$ neutrino superbeam, with the principal objective of measuring $\theta_{13}$. Of course, any existing neutrino beam can exoploit the off-axis trick, and there is a proposal $\left(N O v_{e} A\right)$ to exploit this in the NuMI beam by constructing a new detector off-axis.

More powerful, multiMW neutrino beams are being considered [8]. CERN has discussed a 4 MW 3.5 GeV Superconducting Proton Linac (SPL) [9] and Fermilab is considering [10] a 2MW $8 \mathrm{GeV}$ Superconducting Proton Linac. Both would be multi-function high power proton sources. Such beams could provide better information on $\theta_{13}$ and determine the sign of $\Delta m_{23}^{2}$. With a large detector (a megaton water-Cherenkov), it might be possible to observe CPviolation. Another idea for the creation of neutrino beams of known spectrum and purity is the "beta beam". This topic is covered by the talk of Lindross [12]. 


\section{The Neutrino Factory}

The idea of the neutrino factory is simple [13] - the neutrinos come from the decay of muons in long straight section of a storage ring, directed to a detector hundreds or thousands of kilometres away. This gives simultaneously beams of muon neutrinos and electron antineutrinos (or muon antineutrinos and electron neutrinos) of roughly equal and well-known intensity and spectrum and no background from other neutrino favours. The principal features of a neutrino factory are shown in Figure 2. A suitably shaped and inclined muon storage ring could serve two detectors at different distances, adding significantly to the resolving power of the neutrino factory. For example, the optimum sensitivity to the CP-violating phase $\delta$ is $\sim 3000 \mathrm{~km}$, where matter effects are significant. However, these can be resolved if there is a second detector at either a

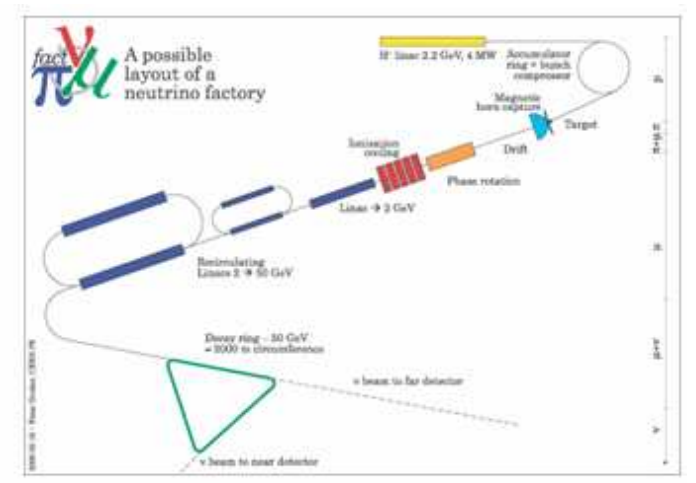

Figure 2 Layout of a Neutrino Factory significantly shorter $(<1000 \mathrm{~km})$ or greater $(>6000 \mathrm{~km})$ distance.

While there are several different schemes for realising a neutrino factory, all share the same basic features. A crucial parameter that drives the design of the neutrino factory is the muon lifetime of $2.2 \mu \mathrm{sec}$, which imposes very significant demands upon the RF systems, although time dilation helps (at $400 \mathrm{MeV}$, the lifetime is already $\sim 10 \mu \mathrm{sec}$, and at $20 \mathrm{GeV}$ it is nearly $0.5 \mathrm{msec}$ ). The main components ar discused below.

A multi-MW proton driver. There are several other applications for high power proton drivers, but the particular feature for the neutrino factory is the very short $(\sim 1 \mathrm{nsec})$ bunch structure. The final stage of the proton driver is an accumulator/compressor ring, which may be fed by either a high-energy linac or a series of rapid-cycling synchrotrons. Critical to the design is the choice of the proton energy. The pion yield is rather flat as a function of proton energy, and the optimum is likely to be a compromise between the cost and complexity of the proton driver (lower energy) and that of the target (higher energy).

Target and pion capture. Multi-MW targets are a new domain. There are broadly two options - liquid metal (mercury) and cooled solid metal. There is a need for greater theoretical understanding, and empirical work, on the behaviour of materials under extreme shock. There have been studies of high intensity proton bunches on liquid mercury, on the behaviour of liquid mercury jets in magnetic fields, and on the resilience of metals under shocks. Solid target configurations include radiation cooled rotating rings and liquid cooled metal beads. The pions, widely distributed in angle and energy, need to be collected, focussed, and sign-selected. There are two schemes - magnetic horns and large open solenoids. The geometry for both is highly constrained, and is intimately related to the target design.

Decay, phase rotation and cooling. The pions decay downstream of the target, after which they are phase rotated to reduce the energy dispersion by decelerating the early (higher energy) muons and accelerating the later (lower energy) muons. At the end of the phase rotation, the peak of the muon energy distribution is around $200 \mathrm{MeV}$, with a dispersion of $\pm 10 \%$. The 
emittance is still too large for conventional acceleration to the final energy (10-50 GeV), although this might be acceptable for a FFAG-based machine. Most designs include a cooling section to compress still further the emittance of the beam. Conventional cooling techniques are too slow. Ionisation cooling, in which energy lost through ionisation is replaced longitudinally through RF acceleration, is sufficiently fast. There is also a heating term coming from the multiple scattering, so that the performance of a cooling channel is critically dependent upon the balance. While ionisation cooling clearly works, it is essential that the efficiency of ionisation cooling is understood. A Muon Ionisation Cooling Experiment (MICE) is proposed [13] to test these ideas.

Muon acceleration and storage ring. The acceleration of the muons will use multiple stages, and perhaps several technologies (FFAG, RLA...). The cost depends upon the final energy chosen for the storage ring. Because the neutrino cross-section increases linearly with energy in this range, and the scope for non-oscillation physics at detectors close to the storage ring is much greater, a higher energy $(\sim 50 \mathrm{GeV})$ might be preferred. With such a facility, it is possible (in principle) to study the disappearance through oscillation of both electron and muon neutrinos and antineutrinos, and the appearance of electron (muon) and tau neutrinos in muon (electron) neutrino and antineutrino beams, providing the most complete set of measurements of the neutrino oscillations. There will also be an enormous range of conventional neutrino and muon physics possible at such a facility.

\section{Summary}

The experimental observation of neutrino oscillations has provided the first clear evidence for physics "Beyond the Standard Model", and has stimulated an exciting search for new ways of creating very high intensity, high purity, high energy, low background neutrino beams. There is a large and active community of accelerator and particle physicists working in this field, whose dedication and work I acknowledge.

\section{References}

[1] See, for example, G. Alterelli, Neutrino 2004, Paris, June 2004.

[2] Adapted from M. Maltoni, T. Schwetz, M.A. Tortola and J.W.F. Valle, hep-ph/04051272 (2004)

[3] A. Aguilar et al, Phys.Rev. D64:112007 (2001)

[4] See, for example, T. Makaya, Neutrino 2004, Paris, June 2004.

[5] See, for example, S Brice, ibid

[6] See, for example, M Thomson, ibid

[7] D. Beavis et al., "Long Baseline Neutrino Oscillation Experiment, E889, Physics Design Report," BNL-52459, (1995)

[8] See, for example, T. Kobayashi, Neutrino 2004, Paris, June 2004.

[9] See, for example, C. Prior, at the "Physics with a MultiMW Proton Source", CERN, May 2004 (http://physicsatmwatt.web.cern.ch/physicsatmwatt/).

[10] See R Garoby, ibid

[11] See, for example, CERN Yellow report CERN-2004-002

[12] M Lindroos, these proceedings

[13] There is a good introduction to neutrino factories by S. Gilardoni, Y. Mori, K. Hanke, R. Edgecock, G Franchetti, and M-G. Catanesi in the ICFA Beam Dyrnamics Newsletter (2002) 29 (http://icfa-usa.jlab.org/archive/newsletter/icfa_bd_nl_29.pdf)

[14] MICE - an International Muon Ionisation Cooling Experiment (spokesperson A Blondel) http://hep04.phys.iit.edu/cooldemo/ 